\title{
A Case of Henoch-Schönlein Purpura with Acute Kidney Injury Accompanied by Severe Gross Hematuria and Erythrocytes-Casts Occlusion in Distal Tubules
}

\author{
Satoshi Matsui, Hiroko Tsuji, Kazuhiro Nagao and Shinji Ono
}

Departments of Nephrology and Hypertension, Mitsubishi Kyoto Hospital, Kyoto, Japan

Correspondence should be addressed to: Satoshi Matsui; smastui-kob@umin.ac.jp

Received Date: 19 April 2014; Accepted Date: 8 May 2014; Published Date: 28 June 2014

Academic Editor: Yao-Hsu Yang

Copyright (C) 2014 Satoshi Matsui, Hiroko Tsuji, Kazuhiro Nagao and Shinji Ono. Distributed under Creative Commons CC-BY 3.0

\begin{abstract}
A 77-year-old man came to our hospital with purpura on lower extremities, severe gross hematuria, and acute kidney injury (AKI). On presentation, he was generally edematous and had no oliguria. Any signs of volume depletion were not detected. After the diagnosis of Henoch-Schönlein purpura (HSP) was confirmed, we initiated hydration and daily oral administration of prednisolone (PSL) $(0.6 \mathrm{mg} / \mathrm{kg})$. AKI improved gradually, but hematuria and fecal occult blood (FOB) did not disappear and sustained for four weeks. Therefore, we added mizoribine (MZR) (1 mg/kg/day) to oral PSL. Three weeks after MZR was administered, both of hematuria and positive FOB resolved. Renal biopsy specimen, after AKI improved, showed some erythrocytes-casts occlusion in distal tubules, not severe mesangial cell proliferation, crescents, IgA depositions, or vasculitis. These pathological pictures suggest that one of the causes of renal manifestations might be erythrocytes-casts occlusion in distal tubules, which is similar to the pathological findings of warfarin-related nephropathy. In our case, massive gross hematuria was observed and supposed to make numerous erythrocytes-casts in tubules, and it might lead to acute tubular injury and AKI. Following his clinical improvement, we tapered oral prednisolone to a dose of $0.4 \mathrm{mg} / \mathrm{kg} /$ day and discharged him. This case shows that we should consider occlusive erythrocytes-casts as one of the causes of AKI when we treat HSP patients with severe gross hematuria.
\end{abstract}

Keywords: AKI, Henoch-Schönlein purpura, erythrocytes-casts occlusion.

\section{Introduction}

Background

Henoch-Schönlein purpura (HSP) is small vessel vasculitis with IgA-dominant immune complex depositions on affected vessel wall of skin, gastrointestinal tract, and even glomeruli. The case with acute kidney injury (AKI) is typically accompanied by proliferation of glomerular epithelial cells causing crescents and IgA-dominant vessel

Cite this Article as: Satoshi Matsui, Hiroko Tsuji, Kazuhiro Nagao and Shinji Ono (2014), "A Case of Henoch-Schönlein Purpura with Acute Kidney Injury Accompanied by Severe Gross Hematuria and Erythrocytes-Casts Occlusion in Distal Tubules," International Journal of Case Reports in Medicine Vol. 2014 (2014), Article ID 917068, DOI: 10.5171/2014.917068 
wall immune deposits [1]. In our case, however, renal biopsy specimen showed some erythrocytes-casts occlusion in distal tubules, not crescents, severe mesangial cell proliferation, IgA depositions, or vasculitis.

\section{Case Report}

A 77-year-old man came to our hospital with a 3-week history of fever, purpura on his legs, abdominal pain, and gross hematuria. His medical history included hypertension, hyperuricaemia, hyperlipidemia, and angina. $\mathrm{He}$ was administered Allopurinol (100 $\mathrm{mg} /$ day), Aspirin (100 mg/day), and Pravastatin $(10 \mathrm{mg} /$ day), not warfarin. He also had no history of cigarette smoking.

Upon physical examination, his body temperature was $37.3^{\circ} \mathrm{C}, \mathrm{SpO}_{2}$ was $98 \%$ (room air), and BP was 112/52 mmHg. He had no oliguria, and any signs of volume depletion were not detected. He had palpable purpura on his edematous legs. He stated that abdominal pain preceded his purpura and resolved spontaneously.

The estimated urinary protein per day was $4.0 \mathrm{~g}$. The urinary sediment contained a lot of erythrocytes and erythrocytes-casts in high power field (HPF). Fecal occult blood (FOB) test was also strongly positive.

His serological data showed slightly positivity for antinuclear antibody with a speckled immunofluorescence pattern at a titre of 1:40. The patient also demonstrated increased complement component 3 (C3;
$140 \mathrm{mg} / \mathrm{dL}$ ), but was negative for the presence of anti-ssDNA, anti-dsDNA, anti-Sm, anti-GBM, p-ANCA, c-ANCA, anti-SSA, anti-SSB, and anti-cardiolipin antibodies, along with rheumatoid factor and lupus anticoagulant. His serum creatinine level was $6.14 \mathrm{mg} / \mathrm{dL}$, and the serum IgA level was elevated to $464 \mathrm{mg} / \mathrm{dL}$. His XIII factor level was not decreased. Laboratory tests included elevated leukocyte counts $(8820 / \mu \mathrm{L})$ with no eosinophilia, normal platelet counts $(206000 / \mu \mathrm{L})$, depressed haemoglobin level $(8.0 \mathrm{mg} / \mathrm{dL})$, and elevated CRP (9.77 mg/dL).

Chest computed X-ray tomography scan showed no pulmonary involvement. Endoscopic screening of his upper and lower gastrointestinal tracts showed no ulcers, tumors, or malignancy.

As his manifestation was AKI suggestive of HSP with severe renal involvement, we initiated hydration and induction therapy before renal biopsy, with daily doses of oral methylprednisolone $(0.6 \mathrm{mg} / \mathrm{kg} /$ day $)$.

He responded well to our therapy, and creatinine and CRP levels gradually turned to $1.5 \mathrm{mg} / \mathrm{dL}$ and $0.1 \mathrm{mg} / \mathrm{dL}$ for the following three weeks. But hematuria and FOB did not disappear and sustained. Therefore, we added mizoribine (MZR) (1 mg/kg/day) to oral prednisolone (PSL). Three weeks after MZR was administered, both of hematuria and positive FOB resolved. Following his clinical improvement, we tapered oral PSL to a dose of $0.4 \mathrm{mg} / \mathrm{kg} /$ day and discharged him. His clinical course is described in Figure 1. 


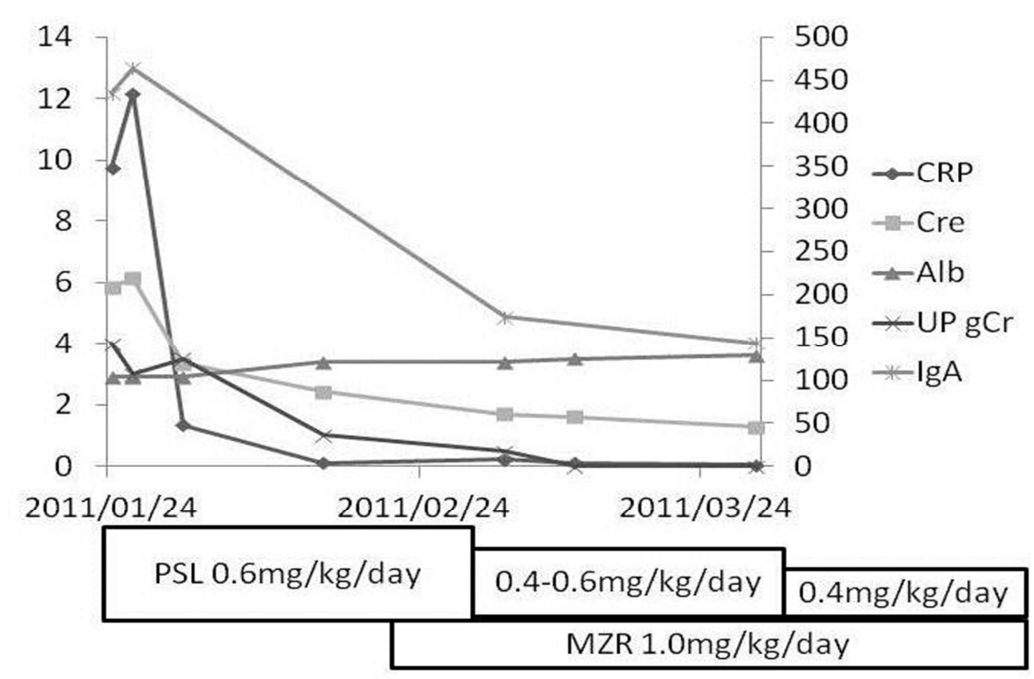

Fig 1.a: Clinical course

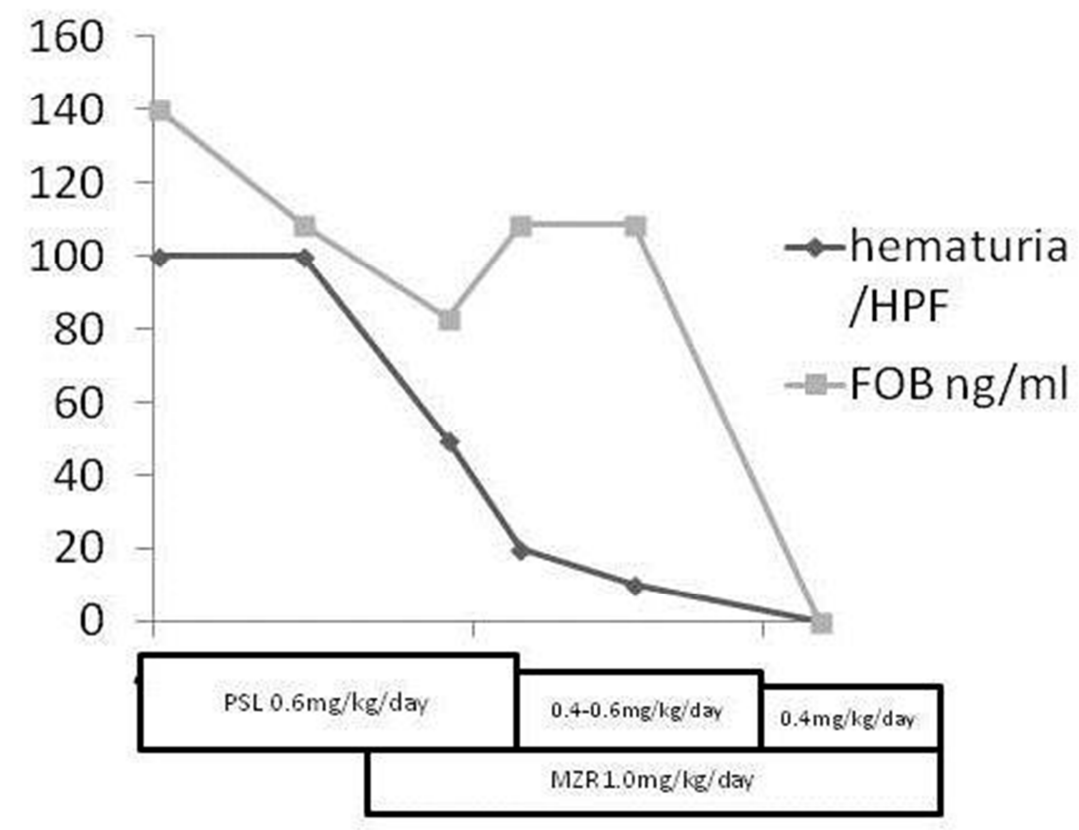

Fig 1.b Course of hematuria and FOB

Fig.1. (a) CRP mg/dL, Cre mg/dL, Alb g/dL, Urinary Protein (UP) g/gCr, IgA mg/dL, (b) Hematuria (/HPF), Fecal Occult Blood (FOB) (ng/ml).

A renal biopsy carried out after his creatinine level decreased to $1.5 \mathrm{mg} / \mathrm{dL}$, and gross hematuria was disappeared, showing the histology of nephrosclerosis and interstitial nephritis. The specimen slide included twenty glomeruli. Of all glomeruli, four were hyalinized and showed global sclerosis. Other glomeruli showed diffuse mild mesangial hypercellularity. One of them accompanied an adhesion of glomerular capillaries to Bowman's capsule, and there was mild tubular atrophy. Since the renal biopsy was performed after renal function had improved, renal histology may be regressed. However, these were not enough changes to cause AKI (Figure 2(a)). Any evidence of vasculitis was not detected. Some erythrocytes-casts were seen in the distal tubules (Figures 2(b) and 2(c)). There were some small foci of lymphoid cell 
accumulation in the hyalinized stroma. Immunofluorescence staining revealed focal slightly mesangial deposition of IgA and C3 (Figure 2(d)). Electron microscopic picture confirmed the proliferation of mesangial cells and the presence of mesangial and paramesangial dense deposits. These pathological pictures suggest that AKI was rather due to erythrocytes-cast occlusion in tubules, not due to severe proliferation of mesangial cells or vasculitis.

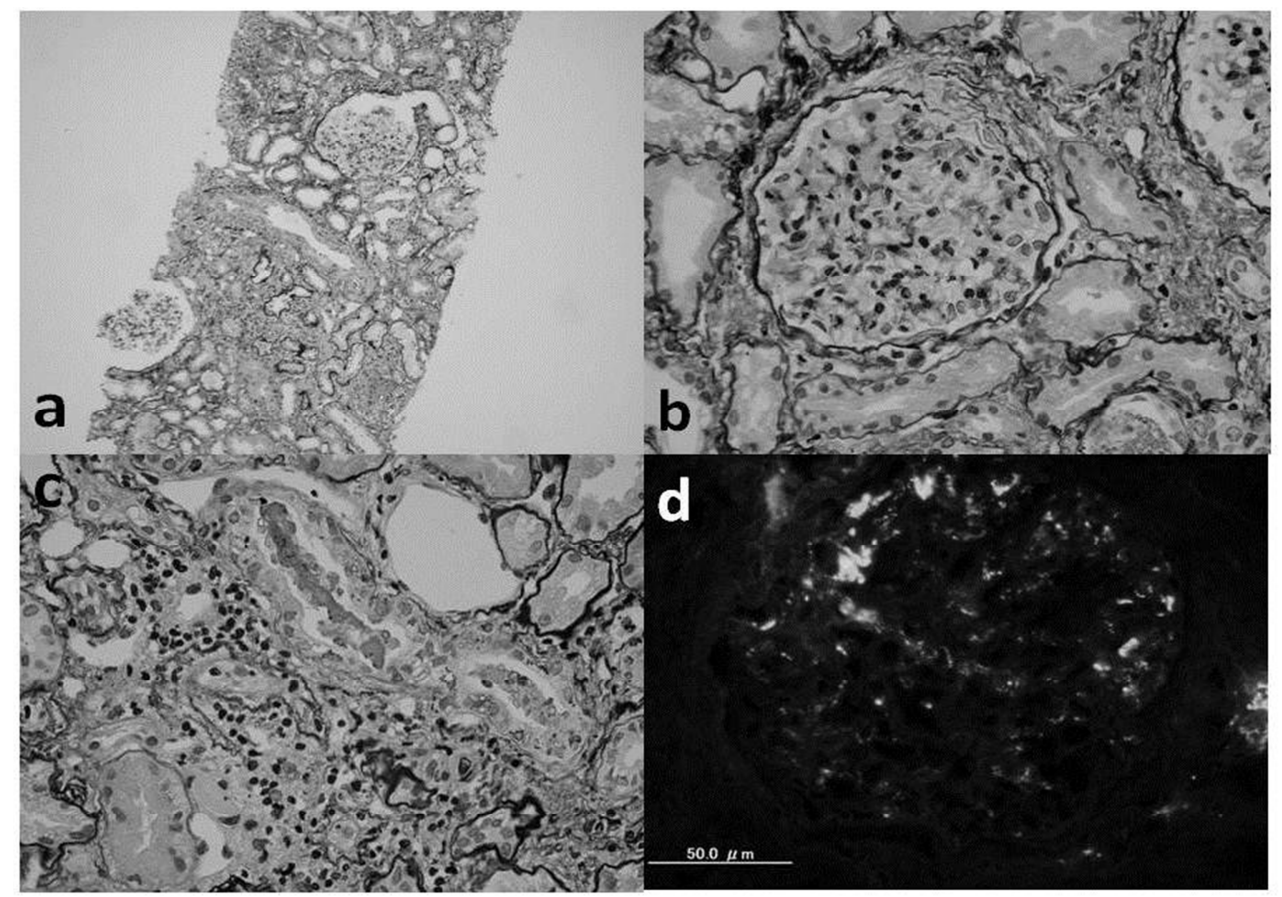

Fig 2 Renal biopsy

Fig.2 (a) Periglomerular Fibrosis and Hyalinosis of Bowman' Capsule (x400, HE) (b), (c) Erythrocytes Casts in the Distal Tubulus. (x400, PAM) (d) Immuno-Fluorescent Pattern: Focal Mesangial Deposition of IgA

\section{Discussion}

About only 5 percent of patients with HSP eventually develop end-stage renal disease, but most of the patients with HSP resolve completely without any therapy [2]. The classifications of both Haas and Lee and colleagues are most widely used, but each has some limitations as a prognostic indicator [3]. As the prognosis is said to be poor when rapidly progressive glomerulonephritis is complicated, and prednisolone and various immunosuppressive agents are often used in the presence of severe renal involvement. Reported immunosuppressive agents include cyclosporine, cyclophosphamide, and azathioprine. IVIG and plasmapheresis have been also reported [1]. However, no standard regimen has been established. These facts may suggest that there are various causes of AKI in HSP and the renal prognosis depends on its underlying etiologies.

Combination of prednisolone and MZR is considered as one of the standard therapies of lupus nephritis and nephrotic syndrome. In such cases, prednisolone is often used as an anti-inflammatory drug and MZR as an immunosuppressive agent. The characteristics of MZR are the lack of oncogenicity and the association with a low incidence of myelosuppression and hepatotoxicity [4]. Recently, Nishioka et al. have reported the usefulness of MZR in maintenance therapy of ANCA-related 
vasculitis [5]. Two cases of HSP successfully treated by PSL and MZR have been also reported [6, 7]. Additive MZR administration in our case might decrease his circulating and depositing IgA effectively and improve sustained hematuria and fecal occult blood. We need to acknowledge the possibility of remission in natural course. However, clinical improvement and time course of hematuria and fecal occult blood after the administration of MZR was very suggestive (Figure 1(b)).

According to the literature, the histopathology of HSP nephritis is that of IgA nephropathy. It is said that the prognostic factors associated with acute kidney injury include massive cellular crescent formation. In our case, we could not find any cellular crescent formation or severe IgA depositions on mesangial cells and capillary loops. Several hyalinized glomeruli and Bowman's capsules might be a ruin of cured crescent, but, usually, healing process is not occurred so early. And besides, we could not find any residue of vasculitis in the stroma, but some small foci of lymphoid cell accumulation in the hyalinized stroma. On the other hand, there were some erythrocyte-casts in distal tubules, which suggested a remnant of macrohematuria of glomerular origin. The pathological findings rather suggest erythrocyte-casts occlusion in distal tubules, than severe mesangial cell proliferation, IgA depositions, or vasculitis.

Recently, AKI during warfarin therapy has been reported [8]. The warfarin-related nephropathy is associated with acute tubular injury and glomerular hemorrhage, caused by erythrocytes in Bowman space and occlusive erythrocytes-casts in tubules. These pathological findings and occlusive mechanism of AKI are very similar to those of our HSP patient. In our case, massive gross hematuria was supposed to make numerous erythrocytes-casts in tubules and may play important role in the development of his renal manifestation. However, we need to acknowledge that no one knows the precise impact of erythrocytes-casts occlusion on the pathogenesis of AKI in this case. Previously, it had been reported that erythrocyte-casts occlusion or hemoglobin casts in tubules might induce AKI. However, recent studies suggested that the main etiology is rather the direct tubular toxicity of hemoglobin, heme, iron, or other molecules released from erythrocytes. It is said that the heme group of hemoglobin decreases nitric oxide availability, promoting intrarenal vasoconstriction and ischemia. On the other hand, lipid peroxidation and physical obstruction of the renal tubule with hemoglobin precipitation are also promoted by cell-free hemoglobin under acidic conditions [9]. These facts suggest that not the amount of erythrocytes-casts occluded in tubules but the severity of the tubular toxicity produced by erythrocytes-casts is more important. In our case, there were erythrocytes-casts in distal tubules, and some small foci of lymphoid cell accumulation in the hyalinized stroma, which might show the existence of more occluded erythrocytes-casts during AKI in acute phase. Considering these underlying mechanisms, aggressive hydration may be justified to prevent and wash out erythrocyte-casts formation, not only immunosuppressive treatment.

In conclusion, we reported a case of Henoch-Schönlein purpura with acute kidney injury accompanied by severe gross hematuria and erythrocyte-casts occlusion in distal tubules. We should consider occlusive erythrocyte-casts as one of the causes of AKI when we treat HSP patients with severe gross hematuria.

\section{Conflict of Interests}

The authors declare that they have no conflict of interests.

\section{References}

1. Davin, J.- C. (2011). "Henoch-Schönlein Purpura Nephritis: Pathophysiology, Treatment, and Future Strategy," Clinical Journal of the American Society of Nephrology March 2011 6: (3) 679-689.

2. Goldstein, A. R., White, R. H. R., Akuse, R. \& Chantler, C. (1992). "Long-Term Follow-Up of Childhood 
Henoch-Schönlein Nephritis," Lancet 1992 Feb 1; 339(8788): 280-2.

3. Feehally, J. (2001). "Predicting Prognosis in IgA Nephropathy," American Journal of Kidney Diseases 2001 Oct; 38(4): 881-3.

4. Kawasaki, Y. (2009). "Mizoribine: A New Approach in the Treatment of Renal Disease," Clinical and Developmental Immunology 2009: 681482.

5. Nishioka, Y., Horita, Y., Tadokoro, M., Taura, K., Suyama, N., Miyazaki, M. et al. (2006). "Mizoribine Induces Remission of Relapsed ANCA-associated Renal Vasculitis," Nephrology Dialysis Transplantation 2006 Apr; 21(4): 1087-8.

6. Kawasaki, Y., Suyama, K., Matsumoto, A., Takano, K., Hashimoto, K., Suzuki, S. et al. (2007). "Efficacy of Tonsillectomy Plus Methylprednisolone Pulse Therapy for a Child with Henoch-Schoenlein Purpura Nephritis," The Tohoku Journal of Experimental Medicine 2007 Mar; 211(3): 291-5
7. Kawakami, T., Shirai, S. Kimura, K. \& Soma, Y. (2010). "Successful Use of Mizoribine to Treat Recurrent Corticosteroid-Resistant Palpable Purpura in a Patient with Henoch-Schonlein Purpura Nephritis," Archives of Dermatolology 2010 Feb; 146(2):212-3.

8. Brodsky, S. V., Satoskar, A., Chen, J., Nadasdy, G., Eagen, J. W., Hamirani, M. et al. (2009). "Acute Kidney Injury during Warfarin Therapy Associated with Obstructive Tubular Red Blood Cell Casts: A Report of 9 Cases," American Journal of Kidney Diseases 2009 Dec; 54(6):1121-6.

9. Moreno, J. A., Martín-Cleary, C., Gutiérrez, E., Toldos, O., Blanco-Colio, L. M., Praga, M., Ortiz, A. \& Egido, J. (2012). “AKI Associated with Macroscopic Glomerular Hematuria: Clinical and Pathophysiologic Consequences," Clinical Journal of the American Society of Nephrology 2012 Jan; 7(1):175-84. 